\title{
3 Research S Suare \\ The Etiologies of Isolated Fourth Cranial Nerve Palsy: A 10-Year Review of 158 Cases
}

\section{Apatsa Lekskul}

Mahidol University Ramathibodi Hospital

Wadakarn Wuthisiri

Mahidol University Ramathibodi Hospital

Phantaraporn Tangtammaruk ( $\sim$ pp.phantaraporn@gmail.com )

Mahidol University Ramathibodi Hospital https://orcid.org/0000-0001-7390-1545

\section{Research Article}

Keywords: fourth nerve palsy, superior oblique palsy, etiologies, vertical strabismus

Posted Date: April 15th, 2021

DOI: https://doi.org/10.21203/rs.3.rs-323169/v1

License: (1) This work is licensed under a Creative Commons Attribution 4.0 International License. Read Full License 


\section{Abstract}

Methods

Patients diagnosed with isolated fourth nerve palsy from January 1, 2009, through July 31, 2020 in Ramathibodi Hospital, were included in this retrospective, observational case series. The demographic data of patients, age at presentation, the etiologies of isolated fourth nerve palsy and neuroimaging results (if indicated) were recorded.

Results

We identified 153 unilateral and 5 bilateral cases of isolated fourth nerve palsy. Mean age at presentation was $38.89 \pm 25.71$ years old. Most of the unilateral cases were congenital $(58.17 \%)$, with vasculopathy (27.45\%), intracranial neoplasm (8.5\%) and other etiologies. Trauma with closed head injury was the most common etiology of bilateral cases $(60 \%)$, followed by ruptured arterovenous malformation (20\%) and vasculopathy $(20 \%)$. Twenty-one of the $43(48.84 \%)$ patients with vasculopathy-associated fourth nerve palsy underwent neuroimaging, with normal findings, and all patients' symptoms resolved within 6 months of symptom onset.

\section{Conclusions}

In our series, most of the isolated fourth nerve palsy cases were congenital, followed in frequency by vasculopathy and intracranial tumor, as in many studies. In cases of vasculopathy, the clinical signs and symptoms resolved within 6 months in all cases: observation was sufficient, with no necessity for neuroimaging. However, neuroimaging should be considered in cases with atypical presentations, such as headache, periorbital pain, ataxia with positive cerebellar signs, or if there is rapid progression or no recovery.

\section{Background}

The most common cause of binocular vertical diplopia is fourth cranial nerve palsy, ${ }^{1}$ which can be present in any age group. Various studies ${ }^{2-5}$ have reported different etiologies of isolated fourth nerve palsy, such as congenital anomalies, trauma, vasculopathy, intracranial neoplasm and undetermined causes.

Different etiologies have been described in various populations. Von Noorden et al., ${ }^{2}$ in a surgical cohort study, and Mollan et al., ${ }^{3}$ in a retrospective case series, reported that congenital fourth cranial nerve palsy was the most common etiology followed by trauma; however, Keane ${ }^{4}$ presented an inpatient series in which trauma was the most common cause. Vasculopathy also was a common etiology. Patients who have microvascular risk factors should be closely followed medically; neuroimaging should be performed only when there is progressive clinical diplopia or there is no recovery. ${ }^{3}$ 
Owing to the various common etiologies in different studies, we aimed to determine the etiologies of isolated fourth nerve palsy in our hospital, and also to focus on the necessity of neuroimaging in this disease.

\section{Methods}

This retrospective study used an electronic medical record system to identify all cases of isolated fourth nerve palsy diagnosed from January 1, 2009, through July 31, 2020 at Ramathibodi Hospital, Thailand, a tertiary referral center.

The study protocol was approved by the Human Research Ethics Committee Faculty of Medicine Ramathibodi Hospital, Mahidol University and adhered to the tenets of the Health Insurance Portability and Accountability Act and the Declaration of Helsinki.

\section{Patients}

Unilateral isolated fourth nerve palsy was defined as incomitant hypertropia, underaction of the superior oblique muscle with or without overaction of inferior oblique muscle, positive unilateral head tilt test or excyclotorsion with the double Maddox rod test less than 10 degrees. Fundus photography was performed to confirm affected eye excyclotorsion.

Bilateral isolated fourth nerve palsy was defined as underaction of bilateral superior oblique muscle with or without overaction of bilateral inferior oblique muscle, alternating hypertropia on horizontal gaze or tilt, positive bilateral head tilt test, excyclotorsion with the double Maddox rod test more than 10 degrees or a V-pattern esotropia of greater than 25 prism diopters. Fundus photography was performed to confirm bilateral excyclotorsion.

Exclusion criteria for all patients were: a history of orbital disorders; myasthenia gravis; multiple cranial nerves disorders; and nuclear and supranuclear disorders with other paretic or restrictive causes.

All patients' medical records were reviewed, to confirm their diagnosis, and to identify the medical conditions and etiologies of the isolated fourth nerve palsy. We recorded the following: demographic data, age at presentation, and the etiologies of isolated fourth nerve palsy. Not every case was confirmed the diagnosis by neuroimaging. Atypical presentations of fourth nerve palsy, such as rapidly progressive diplopia, profound headache, ptosis, periocular pain, ataxia with positive cerebellar signs, and painful eye movements were investigated by neuroimaging, and reviewed.

\section{Classification Of Etiologies Of Isolated Cranial Palsy}

Congenital fourth nerve palsy was diagnosed based on the infant's photographs with facial asymmetry, or on a history of vertical misalignment or abnormal head posture, presence of large vertical fusional 
amplitudes (more than 5 prism diopters) ${ }^{6,7}$ and absence of subjective torsion.

Traumatic fourth nerve palsy was defined as the presence of fourth nerve palsy following head trauma.

Vasculopathy was defined as medical hypertension, diabetes mellitus, dyslipidemia, or a combination thereof, measured within 1 year before or after the onset of isolated fourth nerve palsy and requiring management. Laboratory investigations (complete blood count, fasting blood glucose and lipid profile) were performed. Hypertension was diagnosed using the criteria of the Eighth Report of the Joint National Committee on Prevention, Detection, Evaluation and Treatment of High Blood Pressure, that is, systolic or diastolic blood pressure elevated to more than $140 / 90 \mathrm{~mm} \mathrm{Hg}$ on presentation. ${ }^{8}$ Diabetes mellitus was defined using the 2020 American Diabetes Association criteria. ${ }^{9}$ The criterion for a diagnosis of dyslipidemia was the presence of at least one of the following: more than $200 \mathrm{mg} / \mathrm{dL}$ total cholesterol, more than $130 \mathrm{mg} / \mathrm{dl}$ of low-density lipoproteins, or more than $150 \mathrm{mg} / \mathrm{dL}$ of triglycerides. ${ }^{10}$ Patients with vasculopathy were followed, and their diagnosis confirmed, when ocular misalignment resolved within 6 months.

Intracranial neoplasm was diagnosed if the isolated fourth nerve palsy was a direct result of a neoplasm identified based on clinical symptoms and neuroimaging.

Other etiologies in our study included post-neurosurgery causes, arterovenous malformation (AVM), brain aneurysm and hemorrhagic stroke.

\section{Results}

\section{Etiologies}

Our study identified 153 unilateral (96.84\%) and 5 bilateral (3.16\%) cases of isolated fourth nerve palsy (Table 1). We excluded 3 patients who presented at their first visit with superior oblique palsy, and changed over time to having other cranial nerve palsies or ptosis. The diagnosis in each of those cases was confirmed to be myasthenia gravis, based on a positive acetylcholine receptor antibody test. 
Table 1

Etiology of isolated fourth nerve palsies in Ramathibodi Hospital, Thailand

\begin{tabular}{|llll|}
\hline & Unilateral & Bilateral & Totals (\%) \\
\hline Congenital & 89 & 0 & $89(56.33 \%)$ \\
\hline Trauma & 6 & 3 & $9(5.70 \%)$ \\
\hline Vasculopathic & 42 & 1 & $43(27.22 \%)$ \\
- Hypertension & 11 & & \\
- Diabetes mellitus & 4 & & \\
- Dyslipidemia & 4 & & \\
- Combination & 23 & & \\
\hline Intracranial neoplasm & 13 & 0 & $13(8.23 \%)$ \\
\hline Post-neurosurgery & 1 & 0 & $1(0.63 \%)$ \\
\hline Arterovenous malformation and aneurysm & 1 & 1 & $2(1.27 \%)$ \\
\hline Hemorrhagic stroke & 1 & 0 & $1(0.63 \%)$ \\
\hline Totals & 153 & 5 & $158(100 \%)$ \\
\hline
\end{tabular}

Most of unilateral cases were found to be congenital in origin (58.17\%), followed by vasculopathy $(27.45 \%)$ and intracranial neoplasm (8.50\%). Other causes of unilateral isolated fourth nerve palsy were the effects of neurosurgery, AVM and aneurysm, and hemorrhagic stroke, at $0.65 \%$ each.

Bilateral isolated fourth nerve palsy was much less frequently found in our study. The most common cause of bilateral fourth nerve palsy was trauma (60\%), followed by ruptured AVM $(20 \%)$ and vasculopathy $(20 \%)$.

Twenty-one of the 43 (48.84\%) patients with vasculopathy-associated fourth nerve palsy underwent neuroimaging. The results were normal in all cases. The diagnosis for all of the vasculopathy patients was confirmed when the misalignment symptoms resolved within 6 months.

The 13 cases of intracranial neoplasm in our study comprised: 5 meningiomas (38.46\%), 3 schwannomas (23.08\%), 2 brain metastases (15.38\%), 2 pituitary adenomas (15.38\%) and 1 arachnoid cyst $(7.69 \%)$. The atypical presentations in the patients with neoplasms were diplopia with headache $(69.23 \%)$, periorbital pain $(46.15 \%)$, ataxia with positive cerebellar signs $(23.08 \%)$ and conjunctival injection (23.08\%). Of the patients with intracranial neoplasm, $46.15 \%$ also had microvascular risk factors.

\section{Age at presentation}


The mean age at presentation with fourth nerve palsy was $38.9 \pm 25.7$ years. The etiologies varied by age of presentation (Fig. 1). Eighty-nine patients, with a mean age ( \pm standard deviation) of $24.1 \pm 23.0$ years, were classified as congenital cases. For the 9 trauma patients, the mean age was $40.8 \pm 15.4$ years. The 43 vasculopathy patients presented symptoms in the sixth decade (61.3 \pm 10.8 years). The 13 patients with an intracranial neoplasm also presented with symptoms in the sixth decade (60.7 \pm 9.8 years).

\section{Discussion}

Fourth cranial nerve palsy, the most common cause of binocular vertical diplopia, can present in any age group. ${ }^{1}$ Previously reported etiologies of isolated fourth nerve palsy were congenital causes, trauma, vasculopathy, intracranial neoplasm and undetermined causes. ${ }^{1-5}$ Our study, which reported the etiology of isolated fourth nerve palsy at Ramathibodi Hospital, Thailand, demonstrated frequencies of etiologies that were comparable to those in earlier studies, even though some of those studies reported the etiologies of fourth nerve palsy including isolated palsy and fourth nerve palsy in conjunction with other cranial nerve palsies.

Unilateral isolated fourth nerve palsy was much more common than bilateral palsy in our study (96.84\%). Most of the isolated fourth nerve palsy cases in our study were congenital, similar to results in previous studies. ${ }^{2,3}$ Most congenital cases showed the onset of symptoms within the first decade $(42.70 \%)$. However, more than half of such cases reported the onset of presentation in adulthood, which is consistent with another study. ${ }^{11}$ The delayed onset of symptoms is due to decompensation of control over time, and the increase in presbyopia.

The second-most common cause of fourth nerve palsy in some studies $2,3,12,13$ have reported that trauma was more common whereas in our study was vasculopathy, which is the same as in Rush and Younge. ${ }^{14}$ Vasculopathy in our study accounted for $27.22 \%$ of isolated fourth nerve palsy cases, which were associated with hypertension (25.58\%), diabetes mellitus (9.30\%), dyslipidemia (9.30\%) and combined conditions $(55.81 \%)$. Among the vasculopathy patients, $48.84 \%$ had microvascular risk factors, but had indeterminate history and new-onset diplopia suspected to have other life-threatening diseases. Those patients underwent neuroimaging; all had normal findings. All of the patients had recovered from the misalignment symptoms within 6 months.

On the basis of increased early neuroimaging, identification of atypical presentations and close follow-up of all cases in our center, we reported intracranial neoplasm in $8.23 \%$ of cases, which is the same as Rucker's result, ${ }^{5}$ but much more common than in other studies. ${ }^{2-4,11,14}$ Meningioma (38.46\%) was the most common intracranial neoplasm in our study, followed by schwannoma (23.08\%), brain metastasis (15.38\%), pituitary adenoma (15.38\%) and arachnoid cyst (7.69\%). There have been various reports of isolated fourth nerve palsy associated with intracranial neoplasm, with the age of presentation between the fourth and seventh decades. ${ }^{15-19}$ Our patients with vasculopathy and intracranial neoplasm presented with symptoms in the sixth decade. Patients with intracranial neoplasm also had microvascular risk factors (46.15\%). Atypical presentations were common in our patients, with cases of: diplopia with headache $(69.23 \%)$, periorbital pain (46.15\%), ataxia with positive cerebellar signs $(23.08 \%)$ 
and conjunctival injection (23.08\%). Barton ${ }^{20}$ suggested that patients with an atypical presentation, such as a history of pain or progressive diplopia, and clinical symptoms such as proptosis or ptosis, should undergo urgent imaging. Elmalem et al. ${ }^{15}$ found that $23 \%$ of their patients experienced headache as a presenting symptom in cases of fourth nerve schwannoma. In our study, the fourth cranial nerve palsy was accompanied by periorbital pain. Orbital signs such as chemosis, proptosis and conjunctival injection might be the result of lesions occupying the orbit and causing fourth nerve palsy. Therefore, atypical presentations, as mentioned, warrant neuroimaging without delay.

The other cases of isolated fourth palsy in our study included one that was post-neurosurgery, and one each of AVM and brain aneurysm, and hemorrhagic stroke.

Bilateral isolated fourth nerve palsies are reportedly uncommon, ${ }^{21}$ as we also found in our study. Only $3.16 \%$ of the cases in our population were bilateral; among those, closed head trauma was the most common cause $(60 \%)$.

One limitation of this study is the fact that Ramathibodi Hospital is a tertiary eye referral center. Thus, these data may not be entirely representative of the general population. Another limitation is that, because of our retrospective review of medical records, some information may have been incomplete, including histories of trauma.

\section{Conclusions}

The most common cause of isolated fourth nerve palsy at Ramathibodi Hospital was congenital abnormality, followed by vasculopathies, which all resolved within 6 months of symptom onset, and intracranial neoplasm. All neuroimaging performed in the vasculopathy cases was negative. Patients with isolated fourth nerve palsy who are diagnosed with presumed vasculopathy can be observed without the necessity of neuroimaging, but it should be considered, especially, in cases of atypical presentation, such as diplopia with headache, periorbital pain, ataxia with positive cerebellar signs, rapidly progressive symptoms or no recovery. Furthermore, superior oblique palsy can be considered as one of the presentations of myasthenia gravis.

\section{Abbreviations}

AVM

arterovenous malformation

\section{Declarations}

No funding was received for conducting this study.

All authors certify that they have no affiliations with or involvement in any organization or entity with any financial interest or non-financial interest in the subject matter or materials discussed in this manuscript. 
Ethical approval was waived by the Human Research Ethics Committee Faculty of Medicine Ramathibodi Hospital, Mahidol University and adhered to the tenets of the Health Insurance Portability and Accountability Act and the Declaration of Helsinki in view of the retrospective nature of the study and all the procedures being performed were part of the routine care.

\section{References}

1. Bielschowsky A (1935) Lectures on motor anomalies of the eyes. II. Paralysis of individual eye muscles. Arch Ophthalmol 13:33-59

2. Von Noorden GK, Murray E, Wong SY (1986) Superior oblique paralysis. A review of 270 cases. Arch Ophthalmol 104(12):1771-1776

3. Mollan SP, Edwards JH, Price A et al (2009) Aetiology and outcomes of adult superior oblique palsies: a modern series. Eye (Lond) 23(3):640-644

4. Keane JR (1993) Fourth nerve palsy: historical review and study of 215 inpatients. Neurology 43(12):2439-2443

5. Rucker CW (1958) Paralysis of the third, fourth and sixth cranial nerves. Am J Ophthalmol 46:787794

6. von Noorden GK, Campos EC. Binocular Vision and Ocular Motility Theory and Management of Strabismus. 6th Edition. St. Louis, MO: Mosby (2002) p. 168-210

7. Berens C, Losey RR, Hardy LH (1927) Routine examinations of the ocular muscles and nonoperative treatment. Am J Ophthalmol 10:910-918

8. Whelton PK, Williams B (2018) The 2018 European Society of Cardiology/European Society of Hypertension and 2017 American College of Cardiology/American Heart Association blood pressure guidelines: more similar than different. JAMA 320(17):1749-1750

9. American Diabetes Association (2020) 2. Classification and diagnosis of diabetes: standards of medical care in diabetes-2020. Diabetes Care 43(Suppl. 1):S14-S31

10. Halawani AFM, Alahmari ZS, Asiri DA et al (2019) Diagnosis and management of dyslipidemia. Arch Pharma Pract 10(4):67-70

11. Dosunmu EO, Hatt SR, Leske DA, Hodge DO, Holmes JM (2018) Incidence and etiology of presumed fourth cranial nerve palsy: A population-based study. Am J Ophthalmol 185:110-114

12. Ellis FD, Helveston EM (1976) Superior oblique palsy: diagnosis and classification. Int Ophthalmol Clin 16(3):127-135

13. Akagi T, Miyamoto K, Kashii S, Yoshimura N (2008) Cause and prognosis of neurologically isolated third, fourth, or sixth cranial nerve dysfunction in cases of oculomotor palsy. Jpn J Ophthalmol 52(1):32-35

14. Rush JA, Younge BR. Paralysis of cranial nerves IV III, and VI. Cause and prognosis in 1000 cases. Arch Ophthalmol. 1981;99:76-79 
15. Elmalem VI, Younge BR, Biousse V et al (2009) Clinical course and prognosis of trochlear nerve schwannomas. Ophthalmology 16(10):2011-2016

16. Muthukrishnan J, Bharadwaj K, Singh Y (2016) Isolated fourth cranial nerve palsy due to pituitary macroadenoma. Med J Armed Forces India 72(Suppl 1):S67-S69

17. Pagni CA, Canavero S, Vinci V (1990) Left trochlear nerve palsy, unique symptom of an arachnoid cyst of the quadrigeminal plate. Case report. Acta Neurochir (Wien) 105:147-149

18. Slavin ML (1987) Isolated trochlear nerve palsy secondary to cavernous sinus meningioma. Am J Ophthalmol 104(4):433-434

19. Lee SU, Choi JY, Kim HJ, Kim JS (2018) Central trochlear palsy as an isolated finding with metastatic tumor. J Clin Neurol 14(2):254-256

20. Barton JJS (2009) The symptomatic IV nerve palsy. Neuro-Ophthalmol 28:171-178

21. Lee J, Flynn JT (1985) Bilateral superior oblique palsies. Br J Ophthalmol 69:508-513

\section{Figures}

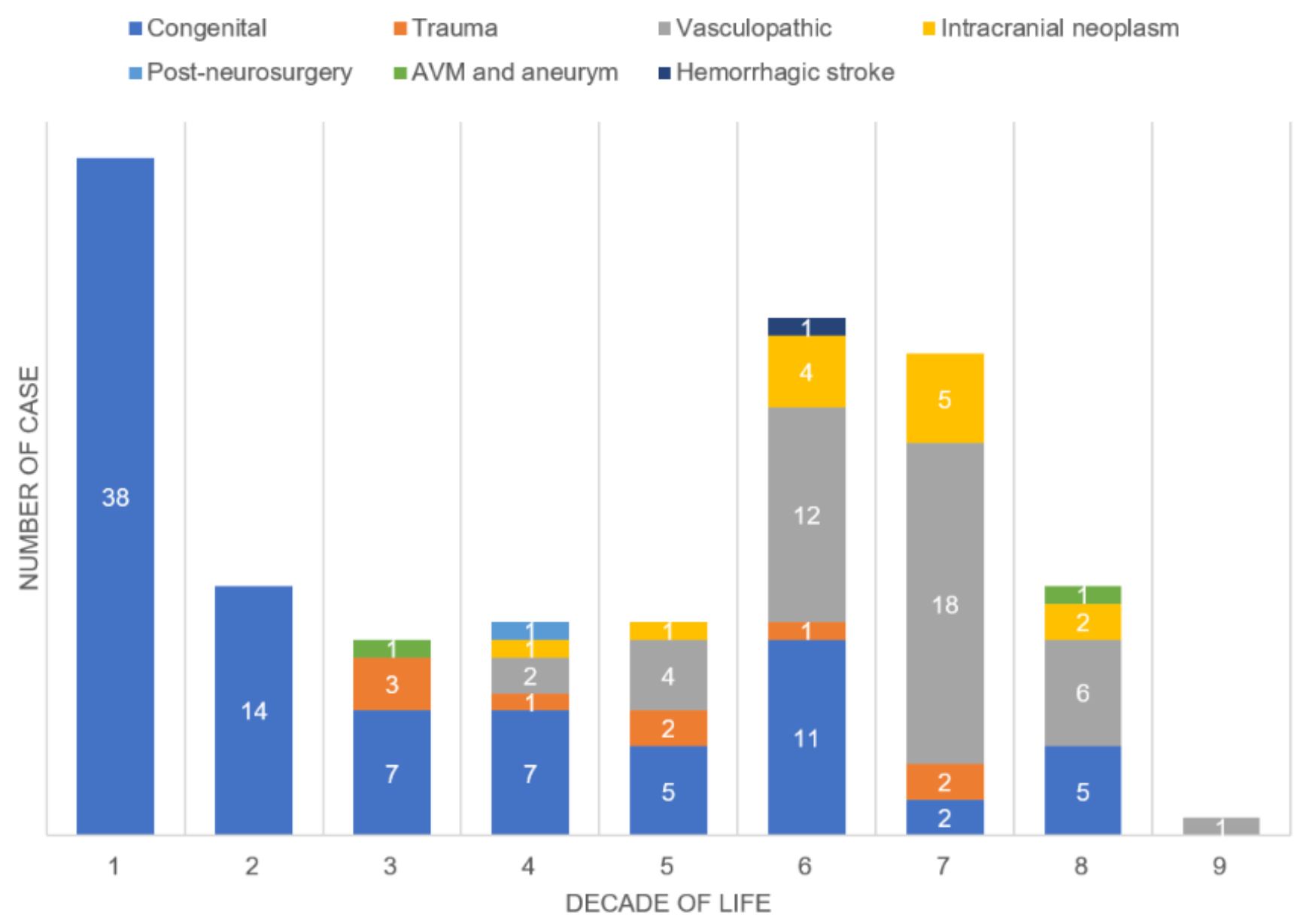

Figure 1 
The etiologies of isolated fourth nerve palsy varied by age of presentation 